\title{
The Rate of Growth of Vapor Bubbles in Superheated Water ${ }^{1}$
}

\author{
By PAUL DERGARABEDIAN,2 PASADENA, CALIF.
}

\begin{abstract}
Calculations are presented for the dynamic stability of vapor and air bubbles in superheated water. These calculations indicate that the values of the bubble radii for which the equilibrium is unstable are restricted to a finite range of radii whose values are governed by the temperature of the water and the initial air content in the bubble. Two theoretical solutions for the rate of growth of these unstable bubbles are considered: (a) Solution of the equation of motion of the bubble radius with the assumption that there is no heat diffusion across the bubble wall; (b) solution which includes the effect of heat diffusion. The two solutions differ appreciably. These two solutions are then compared with the experimental data on the growth of the vapor bubbles in superheated water. This comparison shows agreement with the solution with the effect of heat diffusion included.
\end{abstract}

\section{INTRODUCTION}

$\mathrm{O}$ $\mathrm{NE}$ of the important problems in the field of hydrodynamics today is the occurrence of cavitation in liquids. Cavitation is defined as the coexistence of a vapor or gas phase with the liquid phase. This vapor or gas phase first becomes evident in the form of bubbles distributed throughout the body of the liquid. Of practical significance is the increase in drag experienced by submerged bodies moving through a liquid when cavitation appears; similarly, pumps and turbines operate less efficiently in cavitating flow. The particular phase of the general field of cavitation presented in this paper is the problem of the dynamic stability and rate of growth of these vapor or gas bubbles.

Since the results in the present study are confined to the macroscopic behavior of the bubbles, it suffices to point out some of the present concepts concerning the initial formation of the bubble. The general view $(1)^{3}$ is that bubble formation in cavitating flow, or in boiling, begins from a nucleus within the liquid containing air, or vapor, or both. These gas-phase nuclei are ordinarily submicroscopic in size, and become evident upon the growth of the nuclei through a temperature rise in the liquid or a reduction in the external pressure acting on the liquid.

In dealing with multiple-phase systems the important role of

${ }_{1}^{1}$ This work was supported by the Office of Naval Research under Contract N6onr-24420.

${ }^{2}$ Shell Fellow in Mechanical Engineering at the California Institute of Technology. Present address: U. S. Naval Ordnance Test Station, Pasadena, Calif.

3 Numbers in parentheses refer to the Bibliography at the end of the paper.

Contributed by the Applied Mechanics Division and presented at the Semi-Annual Meeting, Los Angeles, Calif., June 28-July 2, 1953, of The American Society of Mechanical Engineers.

Discussion of this paper should be addressed to the Secretary, ASME, 29 West 39th Street, New York, N. Y., and will be accepted until January 11, 1954, for publication at a later date. Discussion received after the closing date will be returned.

Note: Statements and opinions advanced in papers are to be understood as individual expressions of their authors and not those of the Society. Manuscript received by ASME Applied Mechanics Division, February 11, 1953. Paper No. 53-SA-10. surface phenomena must be considered in the processes involved. Thus in the case of these submicroscopic nuclei the very large forces of surface tension must be overcome to initiate cavitation or boiling. It is well known that degassed pure liquids can withstand very large tensions, or may be superheated considerably, without the formation of bubbles. This effect has been demonstrated by Harvey (2) and subsequently by Pease and Blinks (3). Harvey subjected samples of water saturated with air to pressures of the order of $10,000 \mathrm{psi}$ for several minutes. In this manner the air nuclei are squeezed into solution so that when the solution is brought back to atmospheric pressure, it does not cavitate under the tensions which freely produced cavitation before the pressurization. These same pressure-treated air-water solutions also can be superheated by as much as $80 \mathrm{C}$ without boiling.

For the case of ordinary untreated water the gas-phase nuclei may be stabilized on small solid particles. The presence of a solid, or third phase, is indicated since the surface energy of a bubble bounded by a solid surface and a liquid surface may be very low. Evidence for this condition can be found in the fact that the theoretical boiling point for pure water (4) is much higher than the values obtained by any experiment on superheating of water.

Since the macroscopic behavior of the bubbles formed in a boiling liquid may be considered as entirely analogous to cavitation bubbles, the experiments and calculations in this paper describe the rate of growth of vapor bubbles in superheated water. In the case of boiling liquids by an increase in temperature, the effects upon heat-transfer rates due to the vapor phase are of great interest. The experimental part of this paper is an analysis of high-speed photographs of the growth of vapor bubbles at various degrees of superheat. For the theoretical phase, calculations are presented on the dynamic stability of vapor and air bubbles in order to determine bubble radii for growth. In order to emphasize the important effect of cooling of the bubble wall during the growth, solutions of the equation of motion for the bubble radius are considered both with and without heat conduction across the bubble wall.

\section{Theoretical Formulation of Problem}

Frequent reference is made in the literature on cavitation to Rayleigh's solution for the problem of the collapse of a spherical cavity in a liquid (5). For the present problem of the growth of a bubble the extension of the Rayleigh theory as carried out by Plesset (1) can be used to obtain the equation of motion. The resulting equation is

$$
R \ddot{R}+\frac{3}{2} \dot{R}^{2}=\frac{p_{v}-p_{\infty}+p_{a}-\frac{2 \sigma}{R}}{\rho} \ldots \ldots \ldots
$$

where $\rho$ is the density of the fluid, $R$ is the radius of the bubble at any time $t, \dot{R}=d R / d t, p_{v}$ is the vapor pressure of the water at the appropriate temperature, $p_{a}$ is the partial pressure of air which may be in the bubble of radius $R, p_{\infty}$ is the atmospheric pressure, and $\sigma$ is the surface-tension constant for water.

If the assumption is made that the vapor pressure $p_{v}$ remains 
constant throughout the growth of the bubble, then the bubble growth is isothermal and

$$
p_{a}=p_{a 0} \frac{R_{0}^{3}}{R^{3}}
$$

where $p_{a 0}$ is the initial pressure of the air in a bubble of radius $R_{0}$. Equation [2] implies that no air diffuses across the bubble boundary as it grows. Plesset and Epstein (6) have shown that the diffusion process for gas bubbles is so slow compared to the rate of growth of the bubble that it does not affect the air content of the bubble. Thus Equation [2] is a reasonable expression for the air pressure $p_{a}$ as a function of the bubble radius.

From Equation [1] it can be seen that the bubble is in dynamic equilibrium with the liquid if

$$
\begin{array}{r}
f(R)=\delta p+p_{a 0} \frac{R_{0}^{3}}{R^{3}}-\frac{2 \sigma}{R}=0 \\
\dot{R}=0 \ldots \ldots \ldots \ldots
\end{array}
$$

where

$$
\delta p=p_{v}-p_{\infty}, \quad R_{0}=\frac{2 \sigma}{\delta p+p_{a 0}}
$$

To determine whether the equilibrium of a bubble is dynamically stable or unstable, one may consider $d f(R) / d R$ for the given radius. Then the condition $d f(R) / d R<0$ indicates dynamic stability whereas $d f(R) / d R \geq 0$ is the condition for dynamic instability. Any bubble which is dynamically stable will dissolve through diffusion of air out of the bubble (6); hence the bubbles that need to be considered are the dynamically unstable ones. It is easily seen that the range of equilibrium radii for which $d f(R) / d R \geq 0$ is given by

$$
\frac{4 \sigma}{3 \delta p} \leq R_{0} \leq \frac{2 \sigma}{\delta p}
$$

with

$$
\frac{\delta p}{2} \geq p_{a 0} \geq 0
$$

The solution of Equation [1] is completed as follows: Multiplying by $R^{2} \dot{R}$ and integrating from $R_{0}$ to $R$, Equation [1] becomes

$$
\begin{aligned}
\dot{R}^{2} & =\frac{2}{3} \frac{\delta p}{\rho}-\frac{2 \sigma}{\rho R}+2 \frac{p_{a 0}}{\rho} \frac{R_{0}^{8}}{R^{3}} \log _{\mathrm{e}} R \\
& +\frac{R_{0}^{3} \dot{R}_{0}^{2}+\frac{2 \sigma}{\rho} R_{0}^{2}-\frac{2}{3} \frac{\delta p}{\rho} R_{0}^{3}-2 \frac{p_{a 0}}{\rho} R_{0}^{3} \log _{\mathrm{e}} R_{0}}{R^{3}} \ldots[4
\end{aligned}
$$

Thus

$$
\dot{R}^{2} \sim \frac{2}{3} \frac{\delta p}{\rho}, \quad R \rightarrow \infty
$$

This means that the bubble radius approaches a linear increase with respect to time as $R \rightarrow \infty$ or

$$
R \sim \sqrt{\frac{2}{3} \frac{\delta p}{\rho}} t, \quad R \rightarrow \infty
$$

From Equation [4] it is evident that the terms in $\log _{\mathrm{e}} R / R^{3}$ and $1 / R^{3}$ become small quite rapidly. Physically, this means that the effect of air in a bubble can be important to initiate the growth of the bubble, but its effect upon the subsequent behavior of the bubble radius is negligible; furthermore, all of the initial conditions such as $R_{0}$ and $\dot{R}_{0}$ are involved in the $1 / R^{3}$ term which also vanishes quite rapidly as $R \rightarrow \infty$.

The solution of present interest is the case where $p_{a 0}=0$. Thus Equation [1] becomes

$$
R \ddot{R}+\frac{3}{2} \dot{R}^{2}=\frac{\delta p-\frac{2 \sigma}{R}}{\rho} .
$$

Setting the right-hand side equal to zero, one obtains the equilibrium radius which together with the condition $\dot{R}]_{R}-R_{0}=0$ defines the initial equilibrium of the vapor bubble.

It is convenient to express Equation [5] in dimensionless form. This can be done by the substitutions

$$
u=\frac{\mathrm{R}}{R_{0}}, \quad \tau=\frac{t}{R_{0}} \sqrt{\frac{\delta p}{\rho}}, \quad \dot{u}=\frac{d u}{d \tau}
$$

Equation [5] then becomes

$$
u \ddot{u}+\frac{3}{2} \dot{u}^{2}=1-\frac{1}{u} .
$$

Multiplying Equation [6] by $u^{2} \dot{u}$ and integrating from $u_{i}$ to $u$, where $u_{i}$ is the dimensionless radius at some initial time of $\tau_{i}$, one gets

$$
\dot{u}^{2}=\frac{2}{3}-\frac{1}{u}+\frac{u_{i}{ }^{3} \dot{u}_{i}{ }^{2}-\frac{2}{3} u_{i}{ }^{3}+u_{i}{ }^{2}}{u^{3}}
$$

where $\dot{u}_{i}$ is the velocity of the bubble wall for $\tau=\tau_{i}$. Let $C=u_{i}{ }^{3} \dot{u}_{i}{ }^{2}-2 / 3 u_{i}{ }^{8}+u_{i}{ }^{2}$, where $C$ is a constant taking on vari-

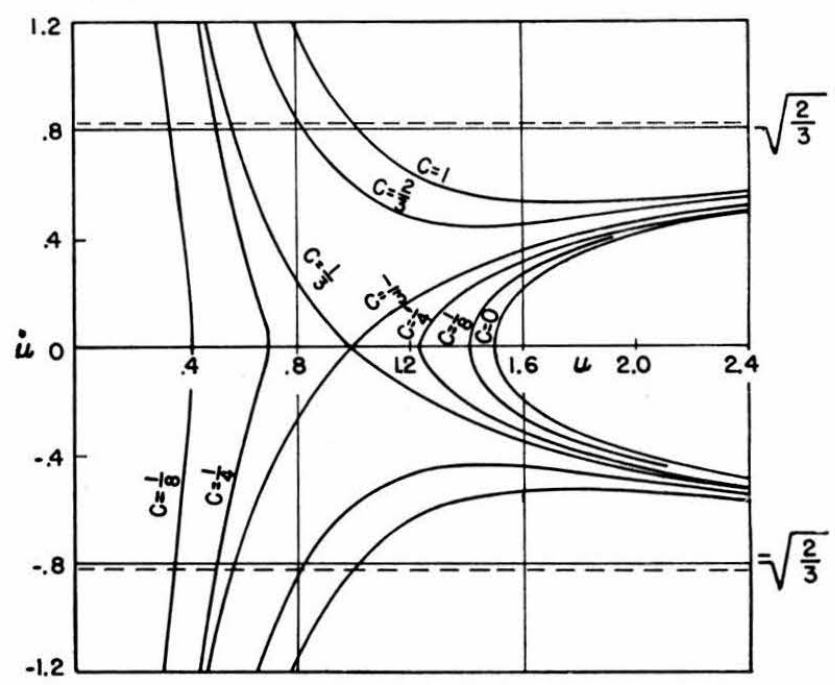

Fig. 1 Dimensionless Velocity $\dot{u}$ for Various Values of $C$ Is Shown as a Function of Dimengionless Radius $u$ as Expressed by Equation [8]

ous values depending upon the values chosen for $u_{i}$ and $u_{i}$. Fig. 1 shows a plot of

$$
\dot{u}= \pm \sqrt{\frac{2}{3}-\frac{1}{u}+\frac{C}{u^{3}}}
$$

for various values of $C$. The significance of the graph is as follows: The choice of a point $(u, \dot{u})$ on the graph as an initial value determines the value of $C$ and hence prescribes the subsequent behavior of the bubble radius as governed by Equation [8]. 
Thus a value of $u_{i}<1$ with $\dot{u}_{i}=0$ means that the bubble will collapse, or a value of $u_{i}>1$ with $\dot{u}_{i}=0$ means that the bubble will grow. The point $u_{i}=1, \dot{u}_{i}=0$ is a singular point since a bubble in this state remains in equilibrium, or loosely speaking, it takes an infinite time for the bubble to increase or decrease in size. However, this equilibrium is dynamically unstable. In the actual physical case such an equilibrium would soon be upset by a slight change in temperature. Taking the positive root of Equation [8] and integrating, one gets

$$
\tau-\tau_{i}^{\prime}=\int_{u_{i}}^{u} \frac{d x}{\sqrt{\frac{2}{3}-\frac{1}{x}+\frac{C}{x^{3}}}}
$$

With the change in variable $y=1 / x$, the integral becomes

$$
\tau-\tau_{i}=\int_{1 / u}^{1 / u_{i}} \frac{d y}{y^{2} \sqrt{\frac{2}{3}-y+C y^{3}}} \cdots
$$

The integral in Equation [9] is an elliptic integral except when $C=1 / 3$ and 0 . For the case $C=1 / 3$, Equation [9] can be expressed as

$$
\tau-\tau_{i}=\sqrt{3} \int_{1 / u}^{1 / u_{i}} \frac{d y}{y^{2}(y-1) \sqrt{y+2}} \ldots \ldots
$$

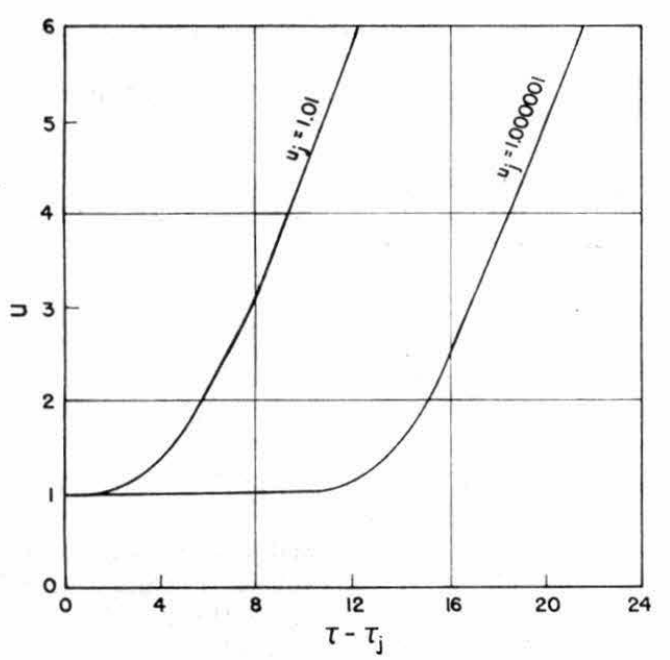

Frg. 2 Dimensionless Bubble Radius $u$ Is Shown for Two Inttial Values of Bubble Radius $u_{j}$ Corregponding to Time $\tau_{j}$ A A A Function of Dimengionless Time $\tau-\tau_{j}$ As Expressed BY Equation [11]

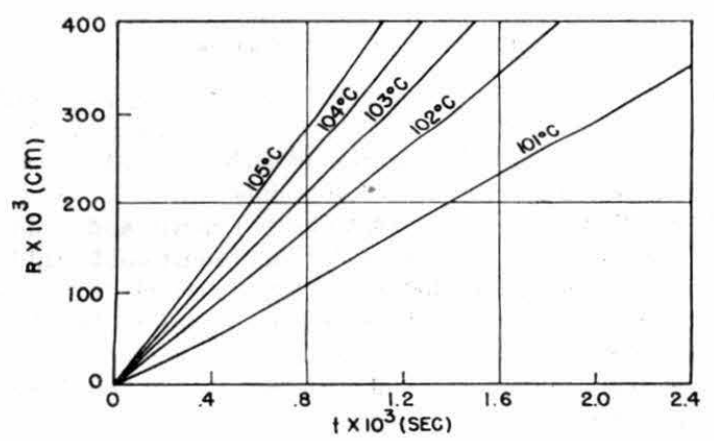

Fig. 3(a) Actual Radius $R$ of Bubble Is Shown for Various Values of Water Temperature $T$ as a Function of the Time $t$, as Obtained From Equation [11]
This corresponds to the case $u_{i}=1, \dot{u}_{i}=0$, in the expression for $C$. The limit here must be $1 / u_{j}<1 / u_{i}$ and $\tau_{j}>\tau_{i}$, since a limit of $1 / u_{i}=1$ means that the integral becomes infinite or the bubble does not grow. The choice of a value $u_{i}>1$ automatically starts the bubble along the trajectory for $C=1 / 3$ at some value $u_{j}$ and $\dot{u}_{j} \neq 0$. The closer one picks $u_{j}$ to 1 , the longer it will take the bubble to grow. The integration of Equation [10] is carried out readily by a separation of the integrand into partial fractions resulting in the following relation between $\tau$ and $u$

$$
\begin{aligned}
& \tau-\tau_{j}=\frac{u}{2} \sqrt{\frac{3}{u}+6}-\frac{u_{i}}{2} \sqrt{\frac{3}{u_{i}}+6} \\
& +\frac{3}{4} \sqrt{\frac{3}{2}} \log _{\mathrm{e}}\left[\frac{\frac{u}{2} \sqrt{\frac{3}{u}+6}+\sqrt{\frac{3}{2}}\left(u+\frac{1}{4}\right)}{\frac{u_{i}}{2} \sqrt{\frac{3}{u_{i}}+6}+\sqrt{\frac{3}{2}}\left(u_{i}+\frac{1}{4}\right)}\right] \\
& -\log _{e}\left\{\left(\frac{u_{j}-1}{u-1}\right)\left[\frac{\frac{u}{2} \sqrt{\frac{3}{u}+6}+\frac{5}{4}\left(u+\frac{1}{5}\right)}{\frac{u_{j}}{2} \sqrt{\frac{3}{u_{i}}+6}+\frac{5}{4}\left(u_{j}+\frac{1}{5}\right)}\right]\right\} \ldots[11]
\end{aligned}
$$

Fig. 2 is a plot of $\tau$ as a function of $u$ as expressed by Equation [11] for $u_{i}=1.01$ and $u_{i}=1.000001$ and shows the effect of taking $u_{j}$ close to $u_{i}=1$. Figs. $3(a)$ and $3(b)$ are plots of the actual radius $R$, as a function of the time $t$, for various liquid temperatures using Equation [11].

The assumption has been made that the vapor pressure has a constant value throughout the growth of the bubble. However, evaporation is a process which proceeds at a finite rate and if this rate is not sufficiently high to keep up with the rate of volume

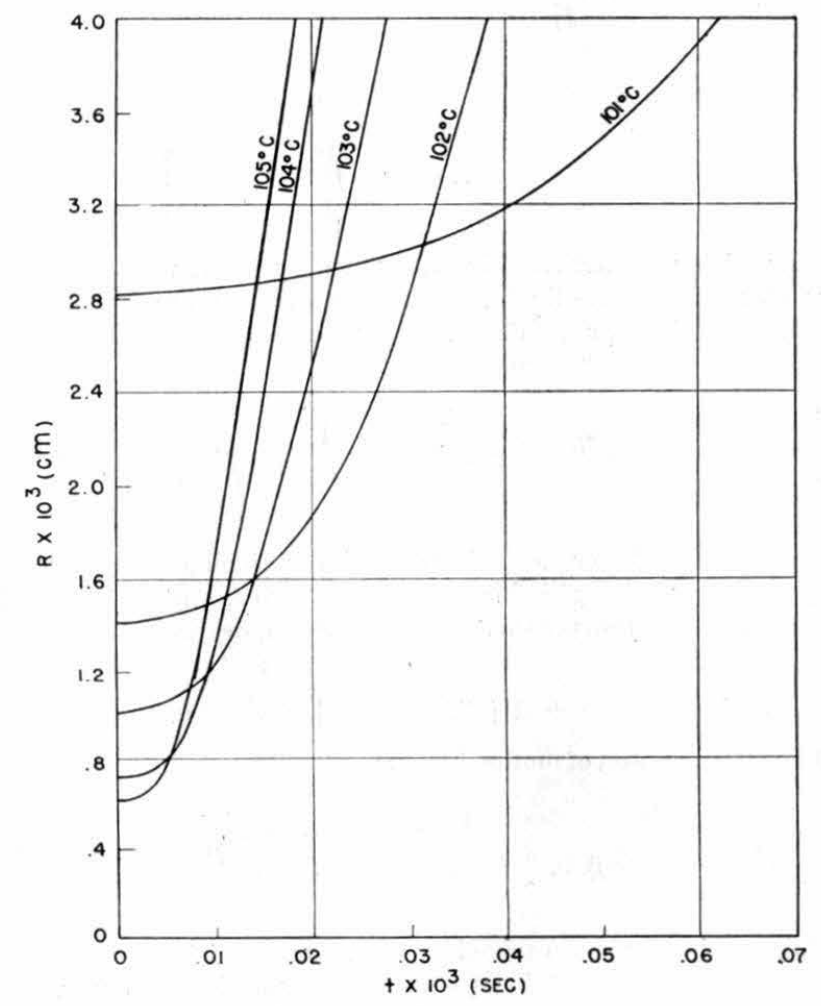

Fig. 3(b) Detailed Plot Is Shown of Fig. 3(a) Near Regton $R=0, t=0$ 
change in the bubble, the vapor in the bubble will behave more like a permanent gas. Plesset tested this assumption for the case of a cavitation bubble (1) with an estimate of evaporation rates based on kinetic theory. Applying a similar analysis here, it turns out that the assumption of $p_{v}$ constant, as far as evaporation rates are concerned, is a reasonable one.

The problem up to this point has been to assume that the bubble expands isothermally; that is, the vapor pressure has been assumed to remain constant throughout the expansion process, having a value corresponding to the bulk temperature of the water. Now this assumption is nearly correct if one thinks of this in terms of the actual variation in the vapor pressure as compared to the absolute initial value of the vapor pressure. However, in terms of the mechanism of bubble growth the variation in vapor pressure has a marked effect on the rate of growth. Heat must be supplied to the bubble to evaporate water and maintain the vapor pressure during growth. This heat is taken out of a water layer surrounding the bubble. Thus the problem as formulated by Plesset and Zwick (7) is to consider the problem of nonsteady heat diffusion which is encountered in dealing with the dynamics of a vapor bubble in a heated liquid. From this problem one obtains the variation of the temperature at the bubble wall as a function of time.

The diffusion problem is solved assuming that the thermal boundary layer is very thin compared to the bubble radius. For the case of the vapor bubble in a liquid, this assumption is made plausible by the fact that not only is the heat capacity much greater in the liquid state than in the vapor state but the thermal diffusivity is about 1000 times smaller. Thus the zeroth-order approximation for the difference between the temperature at the spherical boundary $T(0, t)$, and the initial temperature of the liquid $T_{0}$ is given by (7)

$$
\begin{aligned}
T(0, t)-T_{0}=\frac{D}{K} \eta(t) & -\left(\frac{D}{\pi}\right)^{1 / 2} \\
& \times \int_{0}^{t} \frac{R^{2}(x)\left(\frac{\partial T}{\partial r}\right)}{\left[\int_{x}^{t} R^{4}(y) d y\right]^{1 / 2}} d x
\end{aligned}
$$

where $D$ is the thermal diffusivity of the liquid, $K$ is the thermal conductivity of the liquid, and $\eta(t)$ is the heat source per unit volume in the liquid. For the specific case of the vapor bubble, Equation [12] can be written as

$$
T(0, t)-T_{0}=\frac{D}{K} \eta(t)-\frac{L}{C \sqrt{\pi D}} \frac{\rho^{\prime}}{\rho} \int_{0}^{t} \frac{R^{2}(x) \dot{R}(x)}{\left[\int_{x}^{t} R^{4}(y) d y\right]^{1 / 2}} d x
$$

where $L$ is the latent heat of evaporation of the liquid and $C$ is its specific heat with $\rho^{\prime}$ being the vapor density, and $\rho$ the liquid density. For the present problem the vapor pressure can be expressed as

$$
p_{v}(T)=p_{v}\left(T_{0}\right)+A\left[T(0, t)-T_{0}\right]+B\left[T(0, t)-T_{0}\right]^{2}
$$

Thus the equation of motion becomes

$$
R \ddot{R}+\frac{3}{2} \dot{R}^{2}=\frac{p_{v}(t)-p_{\infty}-\frac{2 \sigma}{R}}{\rho}
$$

This problem has been solved by Plesset and Zwick (8).

A plot of the radius $R$, as a function of the time $t$, is shown in Fig. 4 for a temperature of $103.05 \mathrm{C}$ for the Plesset-Zwick theory and is compared to the solution obtained with the extended

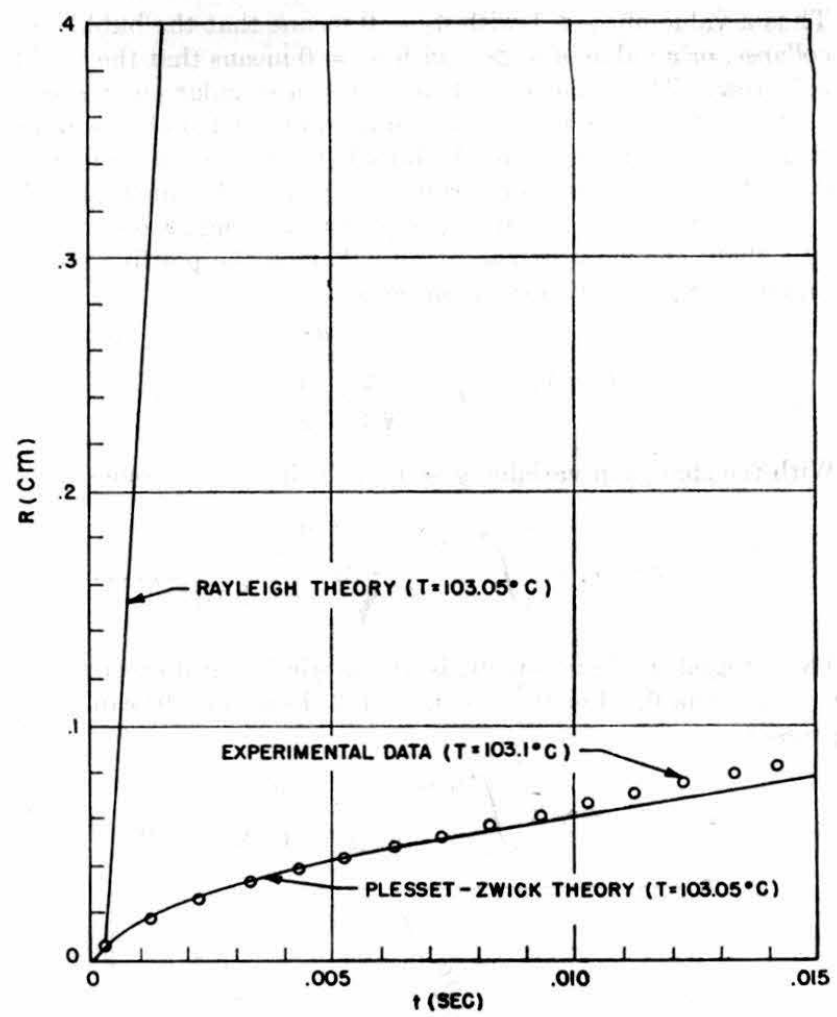

Fig. 4 Bubble Radius Is Shown as a Function of Time for Temperature of 103.1 C as Obtained From Experimental Data (Bubble No. 7), the Rayleigh Theory, and the Plesset-Zwick THEORY

Rayleigh theory for the same temperature. The marked effect that the cooling of the bubble wall has on reducing the rate of growth is apparent. Thus the Rayleigh theory predicts that the bubble approaches a linear rate of growth as the radius increases, the rate being $\dot{R}=\sqrt{2 \delta p / 3 \rho}$, whereas the Plesset-Zwick theory predicts that as the bubble grows the cooling of the bubble wall due to evaporation of the vapor results in the vapor pressure $p_{v}$ approaching the value of the atmospheric pressure $p_{\infty}$, or $\delta p \rightarrow 0$ as $R \rightarrow \infty$; hence $\dot{R} \rightarrow 0$. Consequently a cooling of, say, 2 $\mathrm{C}$ may be small compared to $102 \mathrm{C}$, but in terms of the $2 \mathrm{C}$ of superheat corresponding to the value of $\delta p$, this cooling results in $\delta p \rightarrow 0$. Since the driving force for the bubble growth is produced by $\delta p$, its variation has a pronounced effect upon the rate of growth.

\section{Apparatus and Procedure}

The requirements for obtaining vapor-bubble formation within the body of the liquid at various degrees of superheat are apparent in theory, but the actual realization of such a condition requires a variation in the usual means for boiling water. The requirements are that the liquid must be heated slowly and uniformly, and the walls of the container of the liquid must not be subjected to temperatures so great that a very large thermal gradient exists within a very narrow boundary adjacent to the walls. In addition, these surfaces must be clean and free of pits or scratches. In the case of such gradients all of the bubbles form either within this layer or actually form on the solid surfaces of the container. Thus in the case of heating a beaker of water with a Bunsen burner most of the bubbles form at the bottom of the beaker and quickly rise out of the thermal layer and intermix with the main 
body of the liquid. In this way one has a condition closely approximating the dynamic equilibrium of many large bubbles in the body of the liquid. This condition is realized by the fact that as the bubbles grow they rise into the cooler regions of the liquid above the thermal boundary layer, and their rates of growth are decreased to a point where the bubble can be assumed to be in dynamic equilibrium with the liquid. Since these bubbles are very large, the effects of surface tension are negligible; hence the vapor pressure inside the bubble is very nearly equal to the external pressure on the liquid, which for water heated in a beaker in the laboratory is essentially the atmospheric pressure. Thus the temperature of the boiling water will be very close to that corresponding to the vapor pressure being equal to the atmospheric pressure. For the case of one normal atmosphere of pressure, this temperature will be $100 \mathrm{C}$.

In order to heat the water uniformly the following procedure was used: A beaker was constructed from pyrex tube stock $21 / 2$ in. diam. The tube was cut into a 6 -in. length and a beaker was formed from the tube with a flat face $1^{1 / 4} \mathrm{in}$. wide and 6 in. high. This was done for photographic purposes. The beaker was annealed carefully to make the inner surface very smooth and free from pits. It was cleaned before each experiment with a detergent to remove any oil films present on the surface. The water used in the tests was doubly distilled, and for some of the tests was contaminated with solid impurities such as powdered chalk or sand to produce various degrees of superheat. The water was first boiled in a large container for about $1 \mathrm{hr}$ to remove most of the air in the water. Water boiled in this way contains about 20 per cent of the initial air content. This was not done in the beaker since too much water would evaporate from the test sample. The container with water thus treated was heated by two 250 -watt reflector-type infrared lamps with the beaker located between the lamps.

Fig. 5 shows the arrangement of lamps and beaker. In this way $300 \mathrm{ec}$ of water can be brought to the boiling point in about $10 \mathrm{~min}$. Attempts at obtaining vapor bubbles in the body of the liquid with this technique were completely successful and yielded superheat temperatures up to $107 \mathrm{C}$.

The reasons for the success of this method can be indicated by considering the nature of the radiation of the infrared lamps and the absorption of this radiation by the beaker and the water. The infrared lamp is rated at 250 watts for a line voltage of 115 volts. Under these conditions the tungsten-filament temperature is $2500 \mathrm{~K}$. Since the lamp radiates nearly as a black body, according to factory specifications, Wien's displacement law can be used to obtain the wave length corresponding to the maximum energy output. Thus for $2500 \mathrm{~K}, \lambda_{\max }=1.16 \mu\left(\mu=10^{-4} \mathrm{~cm}\right)$. The distribution corresponding to this temperature shows that approximately 3 per cent of the radiant energy lies between $0.4 \mu$ to $0.7 \mu$ (visible light region). Since the tungsten filament is enclosed by a pyrex bulb, a certain amount of the radiation is absorbed by the bulb itself. Now pyrex is essentially transparent to radiation from $0.3 \mu$ to $3 \mu$, but beyond $3 \mu$ a sheet of pyrex $2 \mathrm{~mm}$ thick absorbs nearly all the radiation. This accounts for about 17 per cent of the total radiated energy originating from the tungsten filament. Thus approximately 80 per cent of the total radiated power is available for heating over a wave band from $0.7 \mu$ to $3 \mu$. Since the beaker itself is pyrex about $2 \mathrm{~mm}$ thick, it will transmit most of the radiation transmitted by the bulb itself. Thus one attains the desired effect of not having the beaker at higher temperatures than the main body of water. The water itself is an excellent absorber of infrared radiation (9) especially from $0.97 \mu$ up to $3 \mu$ which is 68 per cent of the total radiated energy. For example, a layer of water $1 \mathrm{~cm}$ thick absorbs 38 per cent of the radiation of $\lambda=1 \mu$ and 95 per cent of
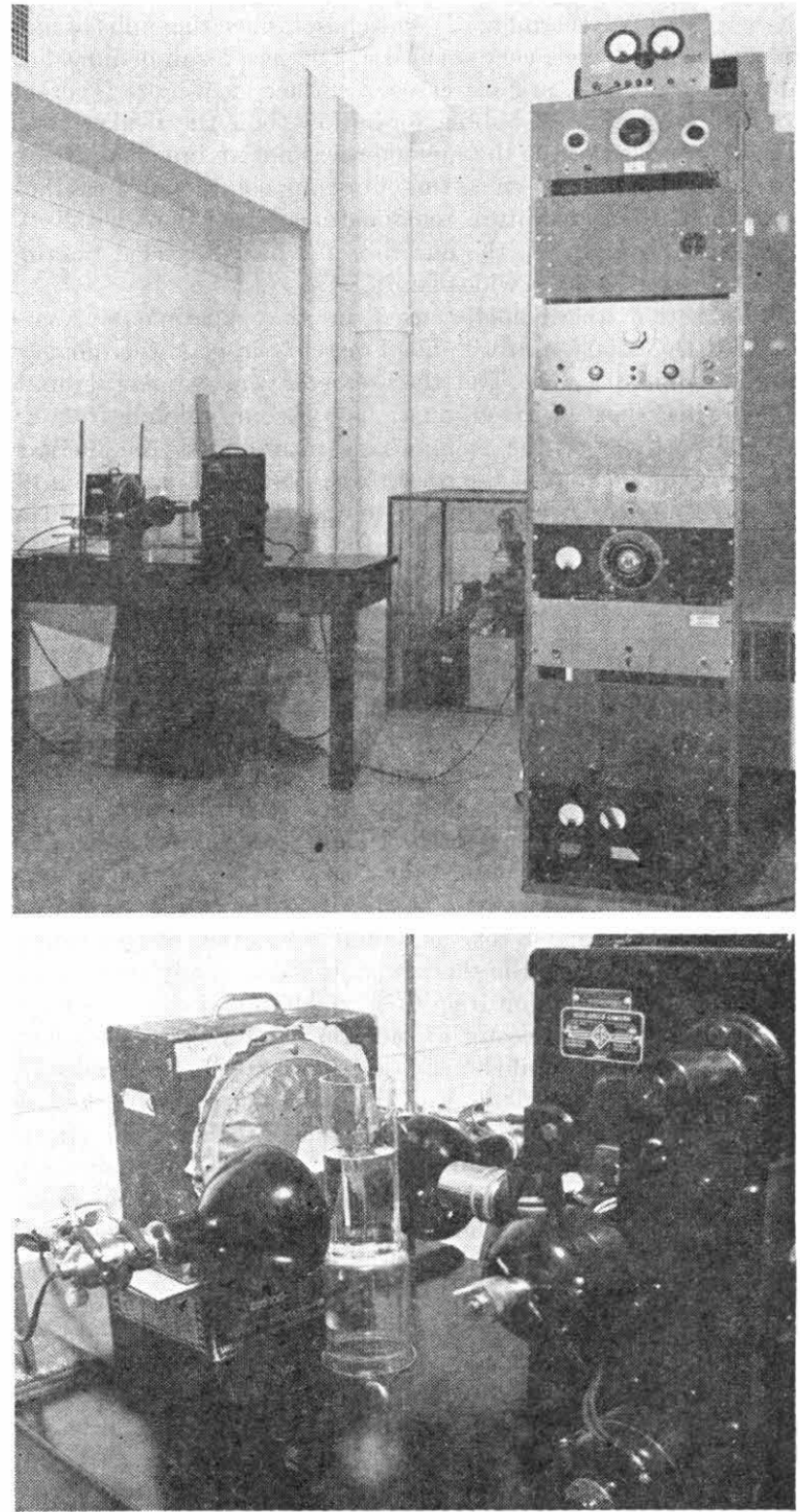

Fig. 5 Upper Figure Shows Entire Experimental Setup of Electronic Equipment, Heat lamps, Camera, and Water Beaker; Lower View is Closeup of Camera, Beaker, Heat LAMPS, AND FLASH LAMP

that at $\lambda=1.4 \mu$ and essentially all the radiation is absorbed in this layer for values of $\lambda>1.4 \mu$. The remaining 12 per cent of the total radiation from $0.7 \mu$ to $0.97 \mu$ is also absorbed but to a lesser extent. The fact that the water is heated very slowly means that the slight thermal gradients existing in the liquid are removed by heat conduction. Thus the temperature of the liquid is raised essentially in a uniform fashion. This is borne out by temperature measurements throughout the bulk of the liquid.

The selection of the temperature-measuring device was controlled primarily by the necessity for having an instrument which could be placed in the water without having bubbles form from the device, since this would eliminate the possibility of having the bubbles form in the body of the liquid. With this restriction, a 
mercury-in-glass thermometer was chosen since the bulbs of most thermometers have a very smooth surface as a result of annealing, and hence provide, in most cases, a surface even more favorable to the prevention of bubble formation than the beaker itself. The thermal lag of the thermometer was not an important factor for these experiments since the only runs considered were those for which the temperature remained nearly constant within 0.1 $\mathrm{C}$ during a given test, the duration of which exceeded the time of any thermal lag by a wide margin.

The actual thermometer used in the experiments was a Braun $100 \mathrm{C}-200 \mathrm{C}$ nitrogen-filled mercury-in-glass thermometer graduated in $0.1 \mathrm{C}$. The thermometer was immersed up to a point just short of the graduations to avoid bubbling from the etched portions of the stem. This meant that the mercury column extended out of the water for all temperatures above 100 $\mathrm{C}$, and thus a correction to the temperature reading would be necessary. Using the correction formula from the Bureau of Standards (10) the maximum correction found necessary was approximately $0.005 \mathrm{C}$. Since this is negligible in terms of $0.1 \mathrm{C}$ accuracy, this correction was neglected.

Another factor affecting the true temperature reading was the fact that the thermometer was not shielded from the effects of radiation. In the discussion on heating water it was shown that the band of infrared radiation from $0.7 \mu$ to $0.97 \mu$ is the least absorbed by the water, and hence this portion of the radiant energy ( 12 per cent of the total) is incident upon the thermometer. It should be pointed out that the thermometer was at all times about $2 \mathrm{~cm}$ from the walls of the beaker. Thus a layer of $2 \mathrm{~cm}$ of water was available for the actual absorption of the radiant energy. The bulb of the thermometer is essentially transparent to the range of radiation from $0.7 \mu$ to $0.97 \mu$ and the mercury in the bulb is a good reflector of radiation and absorbs very little radiation. Thus it could be said that the water acts as a radiation shield for the thermometer, and the radiation effect on the true temperature reading is negligible in terms of the accuracies in the present measurements. To test this, a shield was made of aluminum tubing for the thermometer. The temperature reading for a thermometer without the shield was compared with the reading of the shielded thermometer and the result was that the two simultaneous readings never varied more than $0.1 \mathrm{C}$. The temperature variation throughout the bulk of the liquid was checked after the water had reached a state of boiling, and the variations were found to be much smaller than $0.1 \mathrm{C}$. The accuracy of this type of thermometer is within $\pm 0.1 \mathrm{C}$. Thus considering the two largest errors, namely, the accuracy of the thermometer and the variation in the temperature during a test, the temperature readings obtained are assumed accurate within $0.2 \mathrm{C}$.

The photographic equipment used in this study was of the multiflash type. It consisted of a simple camera in which the recording film moves constantly past the focal plane at a high speed. The camera has no shutter; hence the illumination which was provided by a flash lamp also acted as the shutter. This required that the flash duration be so short that neither the image of the object on the film nor the film itself move an appreciable distance while the light was on. For the present experiments pictures were taken at a rate of 1000 exposures per sec. This rate was found fast enough to record the growth of a vapor bubble and still give a normal-sized $35-\mathrm{mm}$ picture. The camera itself was the standard General Radio type of instrument as shown in Fig. 5, fitted with an $\mathrm{f} 1.5$ Kodak Ektar 2-in. lens. The film used was Eastman 35-mm Background $\mathrm{X}$ and was run through the camera at an average rate of $100 \mathrm{fps}$. The flash lamps used were of the type originally developed by Prof. Harold E. Edgerton and his associates at the Massachusetts Institute of Technology.
Since there is no practical way of knowing just where or when a bubble will form, the chances of catching a bubble on a strip of film $50 \mathrm{ft}$ long which runs through the camera in about $1 / 2$ sec is very small and depends on the area of view and the depth of focus. Thus the choice of magnification depends upon the following factors: It is desirable to have great magnifications since the detail in the pictures of small bubbles will be clearer. However, a factor of 2 in the picture size of the radius of the bubble diminishes the area of view by a factor of 4 and reduces the depth of focus. It was found that magnifications from $\times 0.5$ to $\times 4$ were practical in the sense of the amount of film used and the time involved to obtain a bubble history. This difficulty was coupled with the fact, that, if during a run the temperature variation was too great, even a successful bubbie history from the photographic viewpoint was useless in terms of usible data for the rate of growth. The over-all efficiency of this metnod was to obtain a usable bubble history per $150 \mathrm{ft}$ of film.

With a magnification of $\times 1$ the depth of focus was about $1 / 8$ in. The magnification was determined by placing a scale in the water and focusing on a piece of film in the camera. Thus any bubble in focus could be assumed to have the appropriate magnification corresponding to the setting of the camera since the depth of focus was so small. All of the bubble diameters were measured directly from the negative with a microscopic comparator. Each bubble was measured three times and the average value was taken. The actual measurements of the bubble diameters are estimated to be correct within 3 per cent using this technique.

\section{ANalysis of Data AND TheORY}

Since the bubbles rise through the liquid as they grow, they cannot be called spherical bubbles in the strict sense of the word. As the bubbles rise they tend to assume a shape similar to an oblate spheroid. This effect is very slight during the first part of the growth because the translational motion is very slow compared to the rate of growth of the bubble. During the latter part of the growth the translational effect is very great compared to the rate of growth, and essentially determines the shape of the bubble. Thus for the case of a bubble, the net result is that the dimension of the bubble is reduced in the direction of motion and increased at right angles to the motion. However, the bubble dimensions measured from the negatives were restricted to bubbles occurring during the first part of the growth. Thus the diameter measured in the horizontal plane does not exceed the diameter in the vertical plane by more than 3 per cent (the horizontal measurement was used for the data). The effect of translation on the conduction of heat is not known precisely but presumably is not very large in the present situation. The amount of translation in the bubble histories is less than twice the diameter of the first bubble measured. This can be seen from Fig. 6 which shows an actual bubble history.

The effects of the walls of the container and the proximity of other bubbles were neglected since these distances were large compared to the bubble sizes considered and thus their effects on the bubble growth are probably less than the uncertainty of the data.

Since the time between each picture is $0.001 \mathrm{sec}$, there is an uncertainty in the exact time when the bubble starts to grow from the dynamic equilibrium radius. Thus this time lies between the first visible bubble picture and the frame showing no bubble. Hence one is free to shift the time axis for any bubble history up to the amount of 0.001 sec. This allows one, when comparing bubble histories at a given temperature, to shift the point on the graph \pm 0.001 sec in time to obtain the best fit. In a sense this uncertainty helps in making the comparisons of 


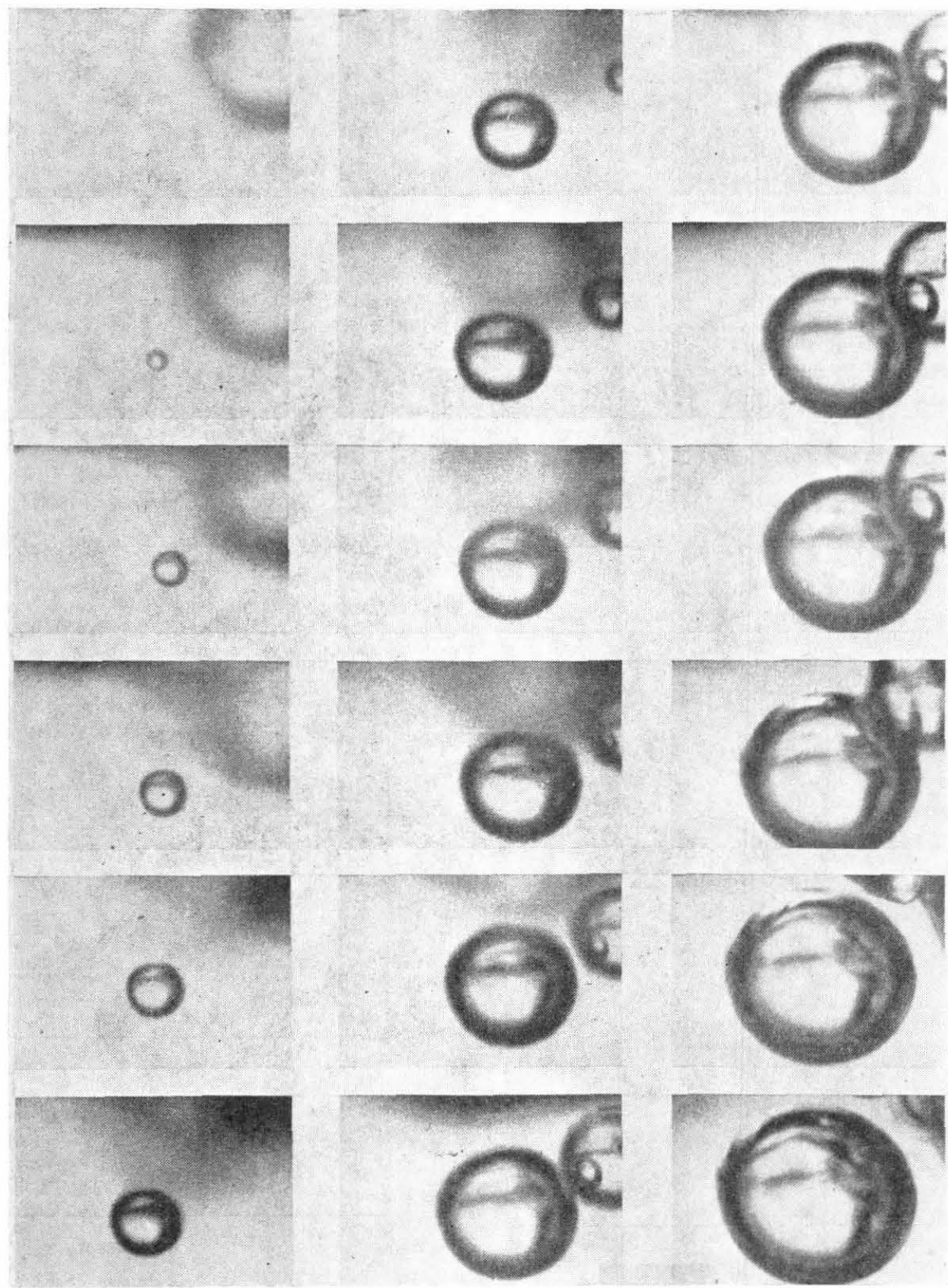

Fig. 6 Photographic History of Rate of Growth of Bubble No. $9(T=103.1 \mathrm{C})$

(Time interval between successive pictures is $0.001 \mathrm{sec}$.)

several bubble histories seem better than they actually may be. However, this does not alter the actual slopes of the radius-time curves.

Since the temperature measurements are estimated to be accurate within $0.2 \mathrm{C}$, the corresponding accuracy of the vapor pressure is required. As an example, the thermal rate of variation of the vapor pressure for the temperature range of 100 $\mathrm{C}$ to $105 \mathrm{C}$ is approximately $4 \times 10^{4} \mathrm{dynes} / \mathrm{cm}^{2} \mathrm{C}$ or for 0.2 $\mathrm{C}$ this gives an error of $8 \times 10^{3} \mathrm{dynes} / \mathrm{cm}^{2}$. Now this error is very small when compared to vapor pressures of the order of $10^{6}$ dynes $/ \mathrm{em}^{2}$ for the temperature range of interest. However, in terms of $\delta p=p_{v}-p_{\infty}$, which is really the important factor for bubble growth, the order of magnitude is $10^{5} \mathrm{dynes} / \mathrm{cm}^{2}$. Therefore the error in $\delta p$ caused by an error of $0.2 \mathrm{C}$ in the thermometer reading is approximately 8 per cent. Since the bubble velocities are roughly proportional to $\sqrt{\delta p / \rho}$, the errors in the general slopes of the radius-time curves obtained from the data should be in error by about 4 per cent from temperature errors alone. This error together with the errors in the negative measurements yields an estimated over-all error in the bubble radii of 10 per cent.

Several bubble histories were obtained for five different temperatures. In some cases all the bubbles for a given temperature were obtained during one run, and for others the histories were 


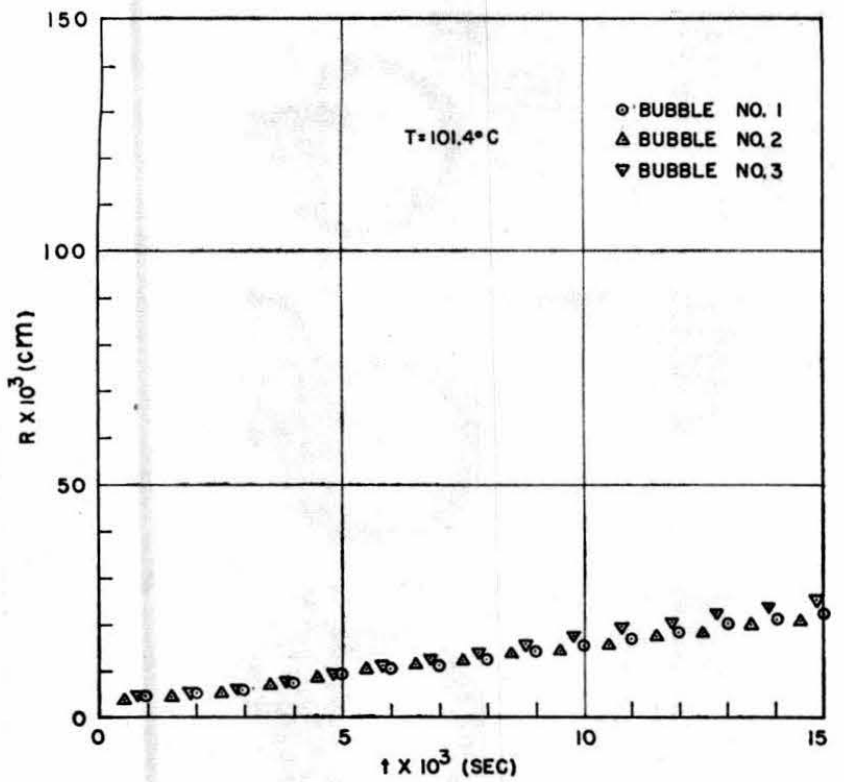

Fig. 7 Radius of Bubble Is Shown as Function of Time for Bubbles Nos. 1, 2, AND 3

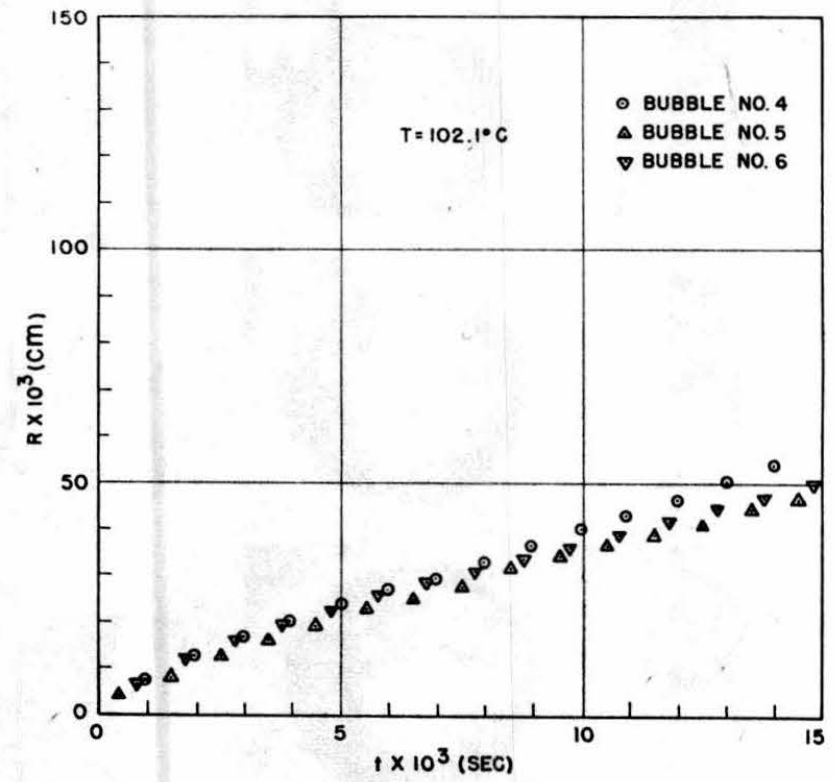

Fig. 8 Radius of Bubble Is Shown as Function of Time for Bubbles Nos, 4, 5, ANd 6

obtained from independent runs. Thus bubbles Nos. 1 and 3 are from one reel and bubble No. 2 from another reel, Fig. 7 . Bubbles Nos. 5 and 6 are from one reel and bubble No. 4 is from another reel, Fig. 8. Bubbles Nos. 7, 8, and 9 are from separate reels, Fig. 9. Bubbles Nos. 11 and 12 are from one reel and bubble No. 10 is from another reel, Fig. 10. Bubbles Nos. 13 and 14 are from the same reel, Fig. 11. For bubble No. 13 two points are missing because the film was blacked out for these two points.

Fig. 4 shows a comparison between the extended Rayleigh theory and the Plesset-Zwick theory with bubble No. 7. From the figure it can be seen that the effect of cooling of the bubble wall has a great effect on the rate of growth. The data follow

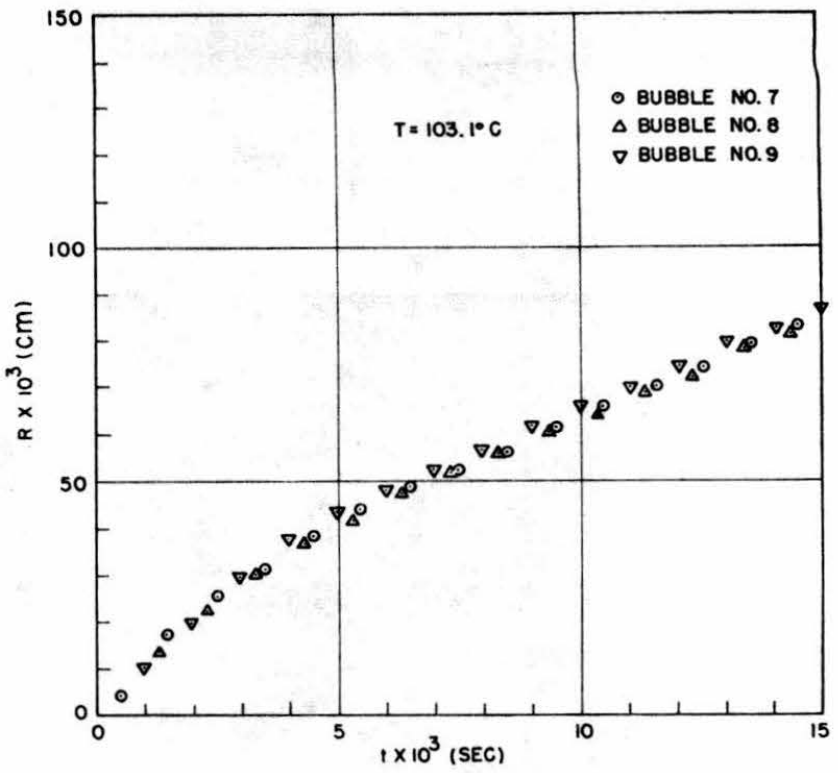

Fig. 9 Radius of Bubble Is Shown as Function of Time for BUbBles Nos. 7, 8, AND 9

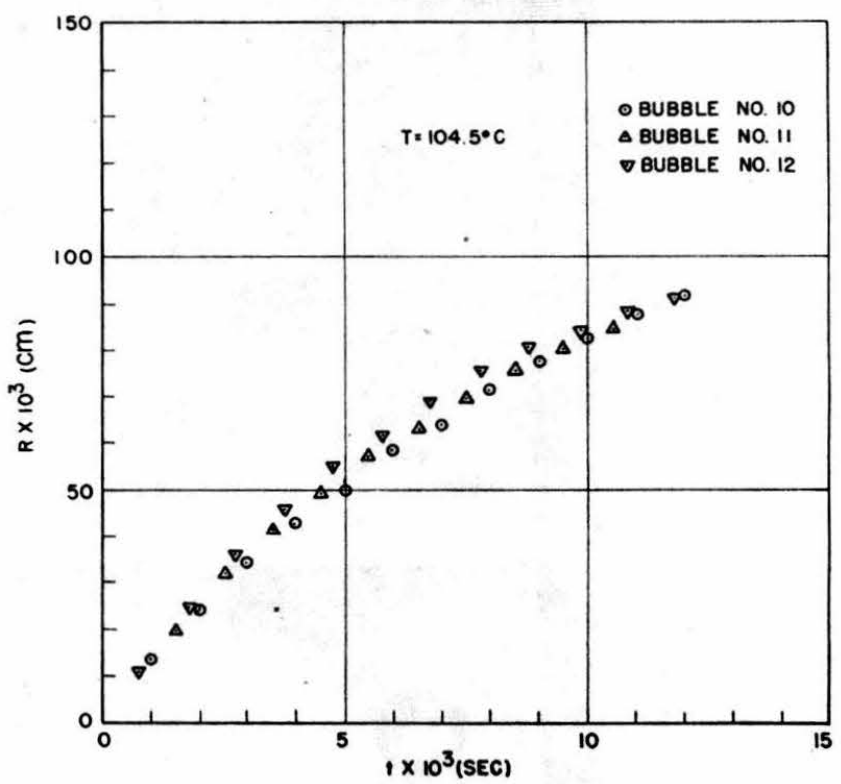

Fig. 10 Radius of Bubble Is Shown as Function of Time for BUBbles Nos. 10,11 , AND 12

the Plesset-Zwick theory very closely as compared to the Rayleigh theory. Thus the cooling of the bubble wall affects the dynamics of the bubble growth to a marked extent.

\section{Acknowledgment}

The author wishes to thank Prof. Milton S. Plesset for suggesting the investigation of the problem, and Dr. Albert T. Ellis for technical aid in setting up the high-speed photographic system. The support of the Shell Fellowship Committee is gratefully acknowledged.

\section{BIBLIOGRAPHY}

1 "The Dynamics of Cavitation Bubbles," by M. S. Plesset, Journal of Applied Mechanics, Trans. ASME, vol. 71, 1949, p. 277. 


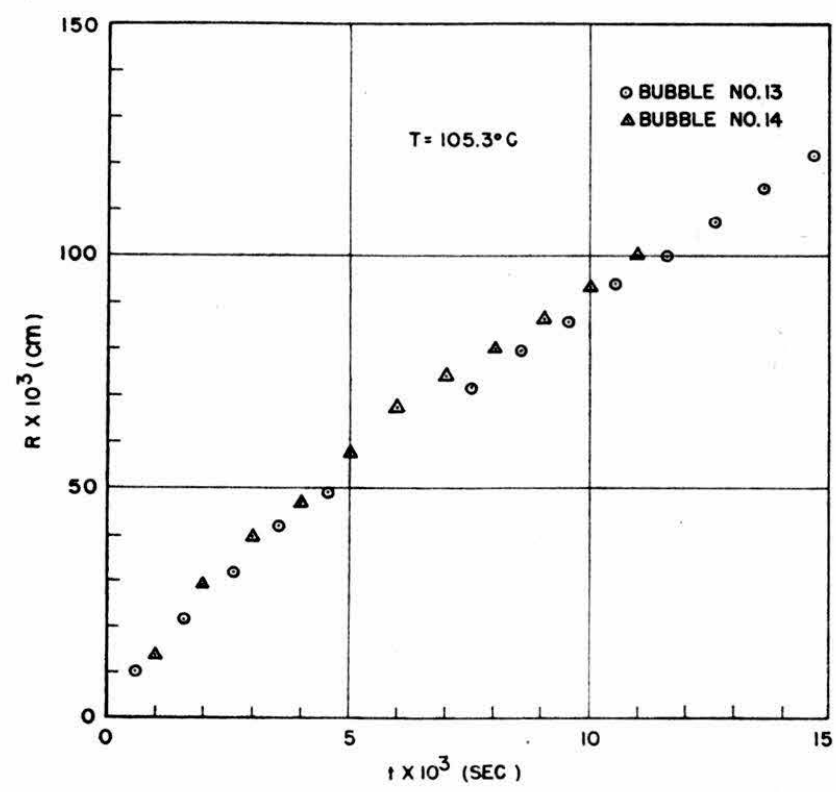

Fig. 11 Radius of Bubble Is Shown as Function of Time for Bubbles Nos. 13 and 14

(The two points missing for bubble No. 13 were the result of faulty negatives.)

2 "On Cavity Formation in Water," by E. Newton Harvey, W. D. McElroy, and A. H. Whitely, Journal of Applied Physics, vol. 18,1947 , p. 162.

3 "Cavitation From Solid Surfaces in the Absence of Gas Nuclei," by D. C. Pease and L. R. Blinks, Journal of Physical and Colloid Chemistry, vol. 51, 1947, p. 556.

4 "Kinetic Theory of Liquids," by J. Frenkel, Oxford University Press, New York, N. Y., and London, England, 1946, p. 372.

5 "On the Pressure Developed in a Liquid During the Collapse of a Spherical Cavity," by Lord Rayleigh, Philosophical Magazine, vol. 34, 1917, pp. 94-98.

6 "On the Stability of Gas Bubbles in Liquid-Gas Solutions," by M. S. Plesset and P. S. Epstein, Journal of Chemical'Physics, vol. 18,1950, p. 1505.

7 "A Nonsteady Heat Diffusion Problem With Spherical Symmetry," by M. S. Plesset and S. A. Zwick, Journal of Applied Physics, vol. 23,1952 , p. 95.

8 To be published, by M. S. Plesset and S. A. Zwick, Californis Institute of Technology, Pasadena, Calif.

9 "Properties of Ordinary Water Substances," by N. E. Dorsey, Reinhold Publishing Corporation, New York, N. Y., 1940, table 160 p. 326.

10 "Temperature-Its Measurement and Control in Science and Industry," by Johanna Busse, American Institute of Physics, Reinhold Publishing Corporation, New York, N. Y., 1941, p. 237. 